\title{
Pre-Exposure Prophylaxis for COVID-19 in Pregnant Women
}

This article was published in the following Dove Press journal:

International Journal of General Medicine

\author{
Melissa C Fesler ${ }^{1,2}$ \\ Raphael B Stricker $\mathbb{D}^{1,2}$
}

'Alan E. Beer Medical Center for Reproductive Immunology, Los Gatos, CA, USA; ${ }^{2}$ Union Square Medical Associates, San Francisco, CA, USA
Correspondence: Raphael B Stricker Union Square Medical Associates, 450 Sutter Street, Suite 1504, San Francisco, CA 94108, USA

Tel + 1 415-399-1035

Email rstricker@usmamed.com

\begin{abstract}
Pregnant women are at higher risk for developing severe complications of COVID-19 including preterm delivery, respiratory failure, and death. Although vaccines to prevent COVID-19 are being developed, pregnant women are not included in the current COVID-19 vaccine trials and initially this population may not be eligible for COVID-19 vaccines due to lack of safety testing in pregnancy. As an alternate approach, we discuss the concept of pre-exposure prophylaxis (PrEP) using medications that are approved for use in pregnant women to prevent gestational problems and severe illness in this high-risk population. In particular, the use of hydroxychloroquine PrEP affords a safe and readily available means to avoid COVID-19 complications in pregnancy.
\end{abstract}

Keywords: COVID-19, SARS-CoV-2, pregnancy, pre-exposure prophylaxis, hydroxychloroquine, vaccine

\section{Introduction}

To date, SARS-CoV-2 has caused more than 96 million cases of COVID-19 and more than two million deaths worldwide. ${ }^{1}$ The United States has witnessed a steady increase in cases, with a recent record of more than 500,000 cases reported in one week of October 2020. Despite such gloomy news, new COVID-19 vaccines have completed or are nearing completion of Phase 3 clinical trials. Although these vaccines may be available to the general population, there is a group of individuals who are likely to be left behind: pregnant women. ${ }^{2,3}$

According to reports from the US Centers for Disease Control and Prevention, pregnant women have a significantly higher risk of severe COVID-19 complications compared to non-pregnant women, including admission to an intensive care unit, need for invasive mechanical ventilation and extracorporeal membrane oxygenation (ECMO), and death. ${ }^{4,5}$ A study from Sweden found that pregnant women with COVID-19 were five times more likely to be admitted to an intensive care unit and four times more likely to receive mechanical ventilation compared to women who are not pregnant. ${ }^{6}$ Risk factors for severe COVID-19 in pregnancy include hypertension, diabetes, cardiovascular disease, cerebrovascular disease, and obesity. $^{7}$

COVID-19 may also affect the fetus. The rate of preterm delivery and stillbirths has increased significantly during the pandemic, and the rate of preterm birth was found to correlate with the severity of maternal disease. ${ }^{8,9}$ Significant placental pathology with fetal demise due to vascular compromise has recently been associated with COVID-19. ${ }^{10,11}$ Because the majority of pregnant women with COVID-19 
reported thus far experienced infection in the third trimester, ongoing surveillance is needed to assess the effect of infections in early pregnancy as well as longer-term outcomes of exposed infants. ${ }^{4,5}$ In a recent study of 594 primarily outpatient pregnant women, SARS-CoV-2 infection was associated with a prolonged disease course during gestation and in the six weeks after pregnancy. ${ }^{12}$

Since pregnant women are typically excluded from vaccine trials including the current ones for COVID-19, it is unlikely that this population will initially receive protection from a novel vaccine due to uncertainty over how it could affect pregnancy., ${ }^{2,3}$ A report of possible cross-reactivity between SARS-Cov-2 spike protein and a component of placental syncytin-1 has led to fears of infertility induced by the COVID-19 vaccines. ${ }^{13}$ Two of the vaccines utilize mRNA technology, a new platform when compared to historical vaccine delivery. Therefore, the effect of this novel technology in pregnant women is unknown and use of these vaccines in pregnancy will require extensive safety testing. Although some medical organizations have speculated that COVID-19 vaccines are safe and effective in pregnancy, clinical support for this speculation is lacking at present. The question then stands: How do we protect this vulnerable population from developing severe COVID-19 symptoms that could cause harm to both the mother and her unborn child? The answer lies in a controversial Food and Drug Administration (FDA)approved medication that has been in clinical use for decades: hydroxychloroquine (HCQ).

\section{History of HCQ}

HCQ is an antimalarial medication that was developed after World War II as a replacement for atabrine, which was given to thousands of soldiers in the South Pacific to prevent the devastating effects of malaria. As an aside, in 1944 the United States produced more than 2.5 billion doses of atabrine, and a similar scale-up of HCQ could be achieved to combat COVID-19. ${ }^{14}$ HCQ has been the preferred drug for malaria prophylaxis due to its excellent safety profile when compared to other medications such as mefloquine, quinine, atovaquone, and chloroquine. ${ }^{15-17}$ It has also been used to treat Q-fever and Babesiosis.

HCQ has been used successfully as an antiinflammatory agent (disease-modifying antirheumatic drug, DMARD) in certain autoimmune and rheumatic conditions such as systemic lupus, rheumatoid arthritis, Sjogren's syndrome, antiphospholipid antibody syndrome, and connective tissue disorders. ${ }^{15,16}$ Despite its recent demonization in the media, HCQ when prescribed correctly and taken as directed is a relatively well tolerated and safe medication, even when taken for extended periods of time. ${ }^{16}$

Multiple studies demonstrate that HCQ has antiviral properties. ${ }^{117}$ Chloroquine analogs such as HCQ have been shown to exhibit non-specific antiviral activity in vitro against viruses such as Ebola, HIV, dengue, chikungunya, Zika, MERS, SARS and influenza, and more recently SARS-CoV-2. ${ }^{16}$ Additionally, HCQ has been shown to protect mouse fetuses from infection with Zika virus, a property that could prove beneficial during the SARS-CoV-2 pandemic. $^{18}$

HCQ is recognized as being safe for use during pregnancy and breastfeeding, and this property is especially important for women in malaria-endemic regions. ${ }^{19-33}$ A study of 133 pregnant women with connective tissue disease found that HCQ was safe when compared to a control group. ${ }^{24}$ Historically, HCQ has been used by rheumatologists and obstetricians to treat women with autoimmune disease to minimize flares and improve pregnancy outcome. Reproductive immunologists have used HCQ to calm overactive immune systems with good outcomes in women who suffer from immunological pregnancy loss and/or failure. ${ }^{19-33}$ Although HCQ has been criticized for prolonging the QT interval, numerous other medications are known to have a greater effect on this EKG parameter without significant clinical concerns, and the safety of HCQ in COVID-19 treatment trials involving nearly 3000 outpatients has recently been confirmed. ${ }^{34-36}$

\section{HCQ Pre-Exposure Prophylaxis (PrEP)}

A crucial aspect of HCQ treatment derives from the distinction between three populations at risk for COVID-19: hospitalized patients who are already infected with SARSCoV-2, people with significant exposure to the virus who receive post-exposure prophylaxis (PEP), and people treated prior to viral exposure who receive pre-exposure prophylaxis (PrEP). HCQ treatment has met with failure in SARS-CoV-2-infected hospitalized patients, and HCQ PEP has also shown variable efficacy. ${ }^{1}$ In contrast, HCQ PrEP prior to exposure or infection has shown positive safety and efficacy results. A recent British study of 120,075 healthcare workers (HCWs) found that these subjects had a 7-8 fold greater risk of developing severe COVID-19 compared to non-HCWs. ${ }^{37}$ With this 
background, four cohort studies of HCWs from India reported positive outcomes for HCQ PrEP. ${ }^{38-41}$ These case-control studies enrolled a total of 2160 high-risk HCWs and demonstrated significantly less SARS-CoV-2 infection in subjects who used HCQ PrEP compared to those who did not. ${ }^{38-41}$

Additional population-based studies in more than 360,000 patients with rheumatic diseases from China, Portugal, and the United States found that HCQ treatment was associated with significantly less SARS-CoV-2 infection. ${ }^{1}$ In a retrospective cohort study of 32,109 rheumatic disease patients from the United States Veterans Administration, the incidence of SARS-CoV-2 infection was extremely low regardless of chronic HCQ use $(0.3 \%$ in users versus $0.4 \%$ in non-users), but mortality was significantly decreased in patients taking HCQ (odds ratio $0.70, \mathrm{p}=0.0031){ }^{1}$

The results of two randomized controlled trials (RCTs) of HCQ PrEP that enrolled 1615 HCWs were recently released, and among 1053 subjects who received HCQ PrEP there were no hospitalizations, no deaths, and no cardiac complications. ${ }^{1}$ In addition, once-weekly dosing appeared to be as effective as twice-weekly or daily dosing for HCQ PrEP. Because the studies were terminated prematurely they were underpowered to show a treatment benefit, however, and additional RCTs of early HCQ treatment for COVID-19 have been recommended. ${ }^{42,43}$ Since RCTs require an average of 5.5 years for completion at an average cost of over a million dollars, it may take a long time to obtain conclusive results from these studies. ${ }^{1}$

\section{HCQ PrEP in Pregnancy}

As noted above, HCQ works well for COVID-19 PrEP prior to infection or exposure to SARS-CoV-2. If HCQ is safe for treatment of pregnant women and effective in prevention of severe disease associated with COVID-19, serious consideration should be given to prescribing HCQ to this vulnerable population that will be neglected upon release of the highly anticipated vaccines. We propose using HCQ at weekly dosing of $400 \mathrm{mg}$ during pregnancy, similar to dosing for malaria prophylaxis. This dose has been used safely in pregnant women, and it should avoid serious disease and maternofetal complications associated with SARS-CoV-2 infection. ${ }^{1,26}$

While RCTs of HCQ PrEP in pregnancy would be desirable, these studies would be difficult to accomplish for several reasons: recruitment for HCQ RCTs has been impeded by negative publicity and political issues surrounding use of the medication in PEP studies and hospitalized patients, and recent HCQ PrEP RCTs have been terminated prematurely due to recruiting failures, as noted above. ${ }^{1}$ In addition, women are reluctant to participate in RCTs during pregnancy due to perception of harm to their unborn children. ${ }^{44}$ Nevertheless, HCQ PrEP RCTs could be attempted in this population in conjunction with appropriate patient education about safety and efficacy of this FDAapproved medication. ${ }^{45}$ In the face of a public health crisis, it is important to consider life-saving approaches based on scientific logic, safety considerations, and clinical availability even if definitive evidence is lacking. ${ }^{1}$

\section{Other Options for Pregnancy PrEP}

Other FDA-approved medications have been touted as potential candidates for COVID-19 treatment. ${ }^{1}$ Unfortunately, most are not considered safe during pregnancy and can cause serious side-effects in pregnant women. Atovaquone, ivermectin, nitazoxanide, tafenoquine, and mefloquine have all been suggested for COVID-19 treatment based on limited in vitro and in vivo studies. Atovaquone and ivermectin have questionable safety during pregnancy (Category $\mathrm{C}$ ) and are therefore not ideal drug candidates for COVID-19 PrEP in pregnant women. ${ }^{46,47}$ Nitazoxanide is safe to take during pregnancy (Category B), but proper dosing remains uncertain for purposes of PrEP. ${ }^{48}$ Tafenoquine is contraindicated in pregnancy due to inadequate human data (no assigned pregnancy category) and potential risk of abortion based on animal studies. ${ }^{49}$ A recent British study found that macrolides taken during pregnancy may be associated with an increased risk of birth defects. ${ }^{50}$

Mefloquine was recently recategorized from a Category $\mathrm{C}$ to a Category $\mathrm{B}$ drug for malaria prophylaxis in pregnancy. ${ }^{51}$ Although this medication shows promise as a potential COVID-19 PrEP candidate, it possesses an unpleasant side effect: psychosis. As such, mefloquine is not advised for those who suffer from depression or any other mental health disease, conditions that are on the rise due to the effect of the COVID-19 pandemic. In contrast, neuropsychiatric complications are extremely rare even in elderly patients taking HCQ for treatment of rheumatic diseases. $^{52}$

\section{Conclusions}

Pregnant women initially may not be eligible to receive COVID-19 vaccines due to the unknown effects on mother and fetus, and these women urgently need an option for protection from COVID-19 when vaccine candidates are 
not available to them. To address this issue, we propose the use of HCQ PrEP to attenuate or prevent SARS-CoV-2 infection in pregnancy. HCQ PrEP should have the same risk-benefit and safety profile in this population as in other high-risk populations. Based on the weight of evidence, HCQ PrEP should be implemented during pregnancy to save lives in the face of an expanding COVID-19 pandemic.

\section{Ethics Statement}

Institutional Review Board approval was not required.

\section{Acknowledgments}

Supported by a grant from the Called2Serve Foundation, Provo, UT. The sponsor had no role in the study design; in the collection, analysis and interpretation of data; in the writing of the manuscript; or in the decision to submit the manuscript for publication. This article is dedicated to the memory of Dr. Gerald Trobough.

\section{Author Contributions}

Both authors meet the authorship requirements of the Committee on Publication Ethics and made significant contributions to the publication. MCF co-conceived the subject, acquired, analyzed and interpreted the original data and citations, and wrote the original draft. RBS coconceived the subject, acquired, analyzed and interpreted additional data and citations, acquired funding and revised the submission for publication. Both authors agreed on the journal to which the article was submitted; reviewed and agreed on all versions of the article before submission and during revision; agreed on the final version accepted for publication and any significant changes introduced at the proofing stage; and agreed to take responsibility and be accountable for the contents of the article.

\section{Disclosure}

RBS is the owner of the Alan E. Beer Medical Center for Reproductive Immunology and reports no other potential conflicts of interest for this work. MCF has no conflicts of interest for this work to declare.

\section{References}

1. Stricker RB, Fesler MC. Flattening the risk: pre-exposure prophylaxis for COVID-19. Infect Drug Resist. 2020;19(13):3689-3694. doi:10. 2147/IDR.S264831

2. Whitehead CL, Walker SP. Consider pregnancy in COVID-19 therapeutic drug and vaccine trials. Lancet. 2020;395(10237):e92. doi:10.1016/S0140-6736(20)31029-1
3. Dashraath P, Nielsen-Saines K, Madhi SA, Baud D. COVID-19 vaccines and neglected pregnancy. Lancet. 2020;396(10252):e22. doi:10.1016/S0140-6736(20)31822-5

4. Zambrano LD, Ellington S, Strid P, et al. Update: characteristics of symptomatic women of reproductive age with laboratory-confirmed SARS-CoV-2 infection by pregnancy status - United States, January 22-October 3, 2020. MMWR Morb Mortal Wkly Rep. 2020;69 (44):1641-1647. doi:10.15585/mmwr.mm6944e3

5. Woodworth KR, Olsen EO, Neelam V, et al. Birth and infant outcomes following laboratory-confirmed SARS-CoV-2 infection in pregnancy - SET-NET, 16 jurisdictions, March 29-October 14, 2020. MMWR Morb Mortal Wkly Rep. 2020;69(44):1635-1640. doi:10.15585/mmwr.mm6944e2

6. Collin J, Byström E, Carnahan A, Ahrne M. Public health agency of Sweden's brief report: pregnant and postpartum women with severe acute respiratory syndrome coronavirus 2 infection in intensive care in Sweden. Acta Obstet Gynecol Scand. 2020;99:819-822. doi:10.1111/aogs.13901

7. DeBolt CA, Bianco A, Limaye MA, et al. Pregnant women with severe or critical COVID-19 have increased composite morbidity compared to non-pregnant matched controls. Am J Obstet Gynecol. 2020. doi:10.1016/j.ajog.2020.11.022

8. Khalil A, von Dadelszen P, Draycott T, Ugwumadu A, O’Brien P, Magee L. Change in the incidence of stillbirth and preterm delivery during the COVID-19 pandemic. JAMA. 2020;324:705-706. doi:10.1001/jama.2020.12746

9. Khoury R, Bernstein PS, Debolt C, et al. Characteristics and outcomes of 241 births to women with severe acute respiratory syndrome coronavirus 2 (SARS-CoV-2) infection at five New York City medical centers. Obstet Gynecol. 2020;136(2):273-282. doi:10.1097/ AOG.0000000000004025

10. Shanes ED, Mithal LB, Otero S, Azad HA, Miller ES, Goldstein JA. Placental pathology in COVID-19. Am J Clin Pathol. 2020;154 (1):23-32. doi:10.1093/ajcp/aqaa089

11. Stonoga ETS, Lanzoni LA, Rebutini PZ, et al. Intrauterine transmission of SARS-CoV-2. Emerg Infect Dis. 2021;27(2). doi:10.3201/ eid2702.203824

12. Afshar Y, Gaw SL, Flaherman VJ, et al. Clinical presentation of Coronavirus disease 2019 (COVID-19) in pregnant and recently pregnant people. Obstet Gynecol. 2020;136(6):1117-1125. doi:10.10 97/AOG.0000000000004178

13. Gong R, Peng X, Kang S, et al. Structural characterization of the fusion core in syncytin, envelope protein of human endogenous retrovirus family W. Biochem Biophys Res Commun. 2005;331 (4):1193-1200. doi:10.1016/j.bbrc.2005.04.032

14. Paltzer S. The other foe: the U.S. Army's fight against malaria in the Pacific theater, 1942-45. The army historical Foundation. 2020. Available from: https://armyhistory.org/the-other-foe-the -u-s-armys-fight-against-malaria-in-the-pacific-theater-1942-45/. Accessed November 20, 2020.

15. Ben-Zvi I, Kivity S, Langevitz P, Shoenfeld Y. Hydroxychloroquine: from malaria to autoimmunity. Clin Rev Allergy Immunol. 2012;42 (2):145-153. doi:10.1007/s12016-010-8243-x

16. Martín-Iglesias D, Artaraz J, Fonollosa A, Ugarte A, Arteagabeitia A, Ruiz-Irastorza G. Evolution of retinal changes measured by optical coherence tomography in the assessment of hydroxychloroquine ocular safety in patients with systemic lupus erythematosus. Lupus. 2019;28(4):555-559. doi:10.1177/09612033 19829826

17. Meo SA, Klonoff DC, Akram J. Efficacy of chloroquine and hydroxychloroquine in the treatment of COVID-19. Eur Rev Med Pharmacol Sci. 2020;24(8):4539-4547. doi:10.26355/eurrev_2020 04_21038

18. Cao B, Sheth MN, Mysorekar IU. To Zika and destroy: an antimalarial drug protects fetuses from Zika infection. Future Microbiol. 2018;13:137-139. doi:10.2217/fmb-2017-0213 
19. Balevic SJ, Green TP, Clowse MEB, et al. Pharmacokinetics of hydroxychloroquine in pregnancies with rheumatic diseases. Clin Pharmacokinet. 2019;58(4):525-533. doi:10.1007/s40262-018-0712-z

20. Bermas BL, Kim SC, Huybrechts K, et al. Trends in use of hydroxychloroquine during pregnancy in systemic lupus erythematosus patients from 2001 to 2015. Lupus. 2018;27(6):1012-1017. doi:10.1177/0961203317749046

21. Buchanan NMM, Toubi E, Khamashta MA, et al. Hydroxychloroquine and lupus pregnancy: review of a series of 36 cases. Ann Rheum Dis. 1996;55(7):486-488. doi:10.1136/ard.55. 7.486

22. Cimaz R, Brucato A, Meregalli E, et al. Electroretinograms of children born to mothers treated with hydroxychloroquine during pregnancy and breast-feeding: comment on the article by Costedoat-Chalumeau et al. Arthritis Rheum. 2004;50 (9):3056-3057. doi:10.1002/art.20648

23. Clowse MEB, Magder L, Witter F, et al. Hydroxychloroquine in lupus pregnancy. Arthritis Rheum. 2006;54(11):3640-3647. doi:10. 1002/art.22159

24. Costedoat-Chalumeau N, Amoura Z, Duhaut P, et al. Safety of hydroxychloroquine in pregnant patients with connective tissue diseases: a study of one hundred thirty-three cases compared with a control group. Arthritis Rheum. 2003;48(11):3207-3211. doi:10. 1002/art.11304

25. Diav-Citrin O, Blyakhman S, Shechtman S, et al. Pregnancy outcome following in utero exposure to hydroxychloroquine: a prospective comparative observational study. Reprod Toxicol. 2013;39:58-62. doi:10.1016/j.reprotox.2013.04.005

26. Irvine M-H, Einarson A, Bozzo P. Prophylactic use of antimalarials during pregnancy. Can Fam Physician. 2011;57(11):1279-1281.

27. Osadchy A, Ratnapalan T, Koren G. Ocular toxicity in children exposed in utero to antimalarial drugs: review of the literature. $J$ Rheumatol. 2011;38(12):2504-2508. doi:10.3899/jrheum.110686

28. Frassi M, Biasini C, Taglieti M, et al. Hydroxychloroquine in pregnant patients with rheumatic disease: a case control observation of 76 treated pregnancies. Lupus. 2004;13(9):755. doi:10.1191/0961203 304lu2018oa

29. Ingster-Moati I, Albuisson E. Visual neurophysiological dysfunction in infants exposed to hydroxychloroquine in utero. Acta Paediatr. 2010;99(1):4; author reply 4-5. doi:10.1111/j.1651-2227.2009.01523.x

30. Kaplan YC, Ozsarfati J, Nickel C, et al. Reproductive outcomes following hydroxychloroquine use for autoimmune diseases: a systematic review and meta-analysis. $\mathrm{Br} J$ Clin Pharmacol. 2016;81(5):835-848. doi:10.1111/bcp.12872

31. Kroese SJ, De Hair MJ, Limper M, et al. Hydroxychloroquine use in lupus patients during pregnancy is associated with longer pregnancy duration in preterm births. J Immunol Res. 2017;2810202.

32. Sammaritano LR, Bermas BL, Chakravarty EE, et al. 2020 American college of rheumatology guideline for the management of reproductive health in rheumatic and musculoskeletal diseases. Arthritis Rheum. 2020;72(4):529-556. doi:10.1002/art.41191

33. Motta M, Tincani A, Faden D, et al. Follow-up of infants exposed to hydroxychloroquine given to mothers during pregnancy and lactation. J Perinatol. 2005;25(2):86-89. doi:10.1038/sj.jp.7211208

34. Costabal FS, Matsuno K, Yao J, Perdikaris P, Kuhl E. Machine learning in drug development: characterizing the effect of 30 drugs on the QT interval using gaussian process regression, sensitivity analysis, and uncertainty quantification. Comput Methods Appl Mech Eng. 2019;1(348):313-333. doi:10.1016/j.cma.2019.01.033

35. Lofgren SM, Nicol MR, Bangdiwala AS, et al. Safety of hydroxychloroquine among outpatient clinical trial participants for COVID-19. Open Forum Infect Dis. 2020;7(11):ofaa500. doi:10.1093/ofid/ofaa500
36. Sogut O, Can MM, Guven R, et al. Safety and efficacy of hydroxychloroquine in 152 outpatients with confirmed COVID-19: a pilot observational study. Am J Emerg Med. 2020;11(40):41-46.

37. Mutambudzi M, Niedwiedz C, Macdonald EB, et al. Occupation and risk of severe COVID-19: prospective cohort study of 120075 UK biobank participants. Occup Environ Med. 2020:oemed-2020106731. doi:10.1136/oemed-2020-106731

38. Chatterjee P, Anand T, Singh KJ, et al. Healthcare workers and SARS-CoV-2 infection in India: a case-control investigation in the time of COVID-19. Indian J Med Res. 2020;151(5):459-467. doi:10.4103/ijmr.IJMR_2234_20

39. Bhattacharya R, Chowdhury S, Mukherjee R, et al. Pre exposure hydroxychloroquine use is associated with reduced COVID19 risk in healthcare workers. MedRxiv. 2020. doi:10.1101/2020.06. 09.20116806

40. Khurana A, Kaushal GP, Gupta R, Verma V, Sharma K, Kohli M. Prevalence and clinical correlates of COVID-19 outbreak among health care workers in a tertiary level hospital in Delhi. MedRxiv. 2020. doi:10.1101/2020.07.21.20159301

41. Goenka MK, Afzalpurkar S, Goenka U, et al. Seroprevalence of COVID-19 amongst health care workers in a tertiary care hospital of a metropolitan city from India. Lancet Reg Health. 2020;3:100041. doi:10.1016/j.lanwpc.2020.100041

42. Wiseman DM, Kory P, Saidi SA, Mazzucco D. Effective post-exposure prophylaxis of covid-19 is associated with use of hydroxychloroquine: prospective re-analysis of a public dataset incorporating novel data. MedRxiv. 2020. doi:10.1101/2020.11. 29.20235218

43. Emani VR, Goswami S, Nandanoor D, et al. Randomised controlled trials for COVID-19: evaluation of optimal randomisation methodologiesneed for data validation of the completed trials and to improve ongoing and future randomised trial designs. Int J Antimicrob Agents. 2020. doi:10.1016/j.ijantimicag.2020.106222

44. Palmer S, Pudwell J, Smith GN, Reid RL. Optimizing participation of pregnant women in clinical trials: factors influencing decisions about participation in medication and vaccine trials. J Obstet Gynaecol Can. 2016;38(10):945-954. doi:10.1016/j.jogc.2016.04.100

45. Taylor MM, Kobeissi L, Kim C, et al. Inclusion of pregnant women in COVID-19 treatment trials: a review and global call to action. Lancet Glob Health. 2020. doi:10.1016/S2214-109X(20)30484-8

46. Mayer RC, Tan KR, Gutman JR. Safety of atovaquone-proguanil during pregnancy. J Travel Med. 2019;26(4):tay138. doi:10.1093/ $\mathrm{jtm} /$ tay 138

47. King CL. Is ivermectin safe in pregnancy? Lancet Glob Health. 2020;8(1):e12-e13. doi:10.1016/S2214-109X(19)30490-5

48. Meneses Calderón J, Figueroa Flores MDR, Paniagua Coria L, et al. Nitazoxanide against COVID-19 in three explorative scenarios. J Infect Dev Ctries. 2020;14(9):982-986. doi:10.3855/jidc.13274

49. Chen V, Daily JP. Tafenoquine: the new kid on the block. Curr Opin Infect Dis. 2019;32(5):407-412. doi:10.1097/QCO.0000000000000574

50. Fan H, Gilbert R, O'Callaghan F, Li L. Associations between macrolide antibiotics prescribing during pregnancy and adverse child outcomes in the UK: population based cohort study. BMJ. 2020;368: m331. doi:10.1136/bmj.m331

51. González R, Pons-Duran C, Piqueras M, Aponte JJ, Ter Kuile FO, Menéndez C. Mefloquine for preventing malaria in pregnant women. Cochrane Database Syst Rev. 2018;11(11):CD011444. doi:10.1002/ 14651858.CD011444.pub3

52. Mascolo A, Berrino PM, Gareri P, et al. Neuropsychiatric clinical manifestations in elderly patients treated with hydroxychloroquine: a review article. Inflammopharmacol. 2018;26:1141-1149. doi:10. 1007/s10787-018-0498-5 


\section{Publish your work in this journal}

The International Journal of General Medicine is an international, peer-reviewed open-access journal that focuses on general and internal medicine, pathogenesis, epidemiology, diagnosis, monitoring and treatment protocols. The journal is characterized by the rapid reporting of reviews, original research and clinical studies across all disease areas. The manuscript management system is completely online and includes a very quick and fair peer-review system, which is all easy to use. Visit http://www.dovepress.com/ testimonials.php to read real quotes from published authors. 